\title{
Molecular Dynamics Simulations of the Collapse of a Cylindrical Pore in the Energetic Material $\alpha-R D X$
}

\author{
Reilly M. Eason ${ }^{1} \cdot$ Thomas D. Sewell $^{1}$
}

Received: 24 July 2015/Accepted: 26 October 2015/Published online: 2 November 2015

(C) Society for Experimental Mechanics, Inc 2015

\begin{abstract}
Molecular dynamics simulations were used to study the shock-induced collapse of cylindrical pores in oriented single crystals of the energetic material $\alpha-1,3,5$ trinitroperhydro-1,3,5-triazine ( $\alpha$-RDX). The shock propagation direction was parallel to the [100] crystal direction and the cylinder axis of the initially $35.0 \mathrm{~nm}$ diameter pore was parallel to [010]. Features of the collapse were studied for Rankine-Hugoniot shock pressures $P_{\mathrm{s}}=9.71,24.00$, and $42.48 \mathrm{GPa}$. Pore collapse for the weak shock is dominated by visco-plastic deformation in which the pore pinches shut without jet formation and with little penetration of the upstream material into the downstream pore wall. For the strong shock the collapse is hydrodynamiclike and results in the formation of a jet that penetrates significantly into the downstream pore wall. Material flow during collapse was characterized by examining the spread and mixing of sets of initially contiguous molecules and evolution of local velocity fields. Local disorder during collapse was assessed using time autocorrelation functions for molecular rotation. Energy deposition and localization was studied using spatial maps of temperature and pressure calculated as functions of time.
\end{abstract}

Keywords RDX · Pore collapse · Shock waves · Molecular dynamics

Thomas D. Sewell

sewellt@missouri.edu

1 Department of Chemistry, University of Missouri-Columbia, Columbia, MO 65211-7600, USA

\section{Introduction}

The role of hot spots in the initiation of detonation in solid energetic materials has been of interest since their existence was proposed by Bowden and Yoffe [1]. Hot spots are, in essence, localized regions of high temperature and pressure in a material that serve as nucleation sites for initiating and possibly sustaining rapid chemistry. Whether a hot spot will grow or quench depends on a sensitive balance between energy release due to local physical and chemical processes and energy transport away from the hot spot due to various dissipation mechanisms [2]. Bowden and Yoffe identified critical hot spots as having sizes of $0.1-10 \mu \mathrm{m}$, lifetimes of $0.1-10 \mathrm{~ms}$, and temperatures greater than $700 \mathrm{~K}$ [1]. Chemical kinetic decomposition models of 1,3,5,7-tetranitroperhydro-1,3,5,7-tetrazocine (HMX) and 1,3,5-triamino2,4,6,-trinitrobenzene (TATB) estimate the critical conditions for hot spots in those materials to be sizes in the $\mu \mathrm{m}$ range and temperatures above $700 \mathrm{~K}$ [3], supporting the values put forth by Bowden and Yoffe.

Accurate determination of hot spot critical parameters is difficult due to the small spatio-temporal scale and difficulty in defining exactly what constitutes a hot spot in a given material under a given loading condition. Field [4] provides a list summarizing works that identify several possible sources of hot spots including pore collapse [5, 6], adiabatic shear bands [7], dislocation pileups [8], and friction [9]. Further insights into the multi-scale interactions between shock waves, defects and interfaces, and the resulting energy localization are essential to forming a more complete understanding of complex detonation initiation processes. Furthermore, the integral role of hot spots in detonation initiation provides strong motivation for understanding the dominant formation mechanisms for a given kind of material and loading scenario. 
The relationship between pore collapse and the generation of hot spots has received considerable attention. High-speed photography used in conjunction with photoluminescence during the collapse of 3-12 mm cavities in $12 \%$ by weight gelatin/water mixtures and ammonium nitrate/sodium nitrate emulsions revealed that the principal ignition mechanism is formation of a hydrodynamic jet that impacts that downstream wall [10-13]. The arrangement of pores relative to each other in space is known to have an effect on a shock wave traveling through a material [14]. Particle image velocimetry measurements of the collapse of $3 \mathrm{~mm}$ diameter cylindrical cavities arranged in various array structures in a mixture of agarose and glycerol gradient buffer showed that for pores aligned parallel to the impact direction the velocity fields exhibited a defracted wave structure from the upstream pore that partially shielded, and slowed, the collapse of the downstream pore. However, when the pores were arranged in a staggered pattern a faster collapse was observed. Dattelbaum et al. [15] mixed solid silica beads and microballoons into liquid nitromethane to act as micron-scale hot spot sources. While both types of nitromethane mixtures were more sensitive compared to the pure liquid, the effect was larger when hollow microballoons were used. This was attributed to the pore collapse that occurred after shock passage. These studies illustrate that the mechanism of collapse, existence of other nearby defects, and composition of a pore all have an effect on the efficiency of energy deposition that occurs upon collapse.

The mm-scale pores mentioned in the preceding paragraph are larger than the ones that exist in a real composite explosive [16-18]. Direct experimental observation of pore collapse in real explosives is extremely difficult due to the high strain rates and small spatio-temporal scales over which pore collapse occurs, and because the phenomena of interest occur in the bulk of an optically impenetrable material. Computer simulations provide a practical approach for elucidating the details of pore collapse with essentially arbitrary spatial and temporal resolution. Hydrocodes are widely applied to continuum-scale problems involving pore collapse. However, care should be taken to ensure that the model description and numerical implementation accurately capture the physics of the material and processes on the spatial scale being studied. As examples, choice of mesh resolution and dissipative mechanisms can affect temperature distribution [19], the method by which strain localization is incorporated determines the amount of heat generated [20], and inclusion of the temperature-dependent liquid viscosity can affect the predicted extent of chemical reaction [21, 22]. Ball et al. [23] used computational fluid dynamics to simulate the experimental setup of Bourne and Field [24]. They obtained agreement between the locations of hot gas predicted by their simulations and the areas of luminescence seen in the Bourne and Field experiment. Tran and Udaykumar [25, 26] simulated pore collapse for both reactive and inert cases in a material with mechanical properties similar to HMX. They reported the pressures, temperatures, and ratios of internal energy to kinetic energy observed during the collapse of a $5 \mu \mathrm{m}$ diameter pore. The formation of a hydrodynamic jet was found to be responsible for the greatest increase in internal energy. Jackson et al. [27] have recently developed a model for which the goal is to reproduce data from sensitivity tests to gain a better understanding of the factors that determine material sensitivity. They used parameters from microscale simulations as input to macro-scale simulations in which shocks produced by the collapse of hot spots were strengthened by interacting with downstream hot spots where ignition had begun but not completed. Most simulations of pore collapse consider idealized spherical pores in 3-D or cylindrical pores with the cylinder axis oriented normal to the shock direction in 2-D. However, Levesque and Vitello [28] analyzed temperature maps obtained from continuum simulations of shocked-induced pore collapse in TATB containing other pore morphologies and determined that higher temperatures are achieved for certain oriented non-spherical pore shapes including elliptical, cylindrical, and conical.

Two limitations of continuum-scale models are the fundamental inability to resolve features that occur on the molecular scale and the associated requirement to formulate and parameterize physical models that effectively capture the sub-scale physical phenomena. At the other extreme, molecular dynamics (MD) simulations can be used to study materials with atomic-scale resolution, but typically only for sub-micron space and sub-nanosecond time scales. (This statement applies to the situation studied here, wherein molecular systems are described using empirical all-atom potential energy functions that include intramolecular flexibility and long-range intermolecular interactions but not chemical reactivity; for reactive potentials the simulation domains are typically much smaller). However, MD simulations can still provide useful information concerning the mechanisms involved in nanoscale pore collapse processes. For example, Holian et al. [29], employing MD simulations of ejecta release and recompression across a planar gap in a Lennard-Jones system, determined that vaporization of material into the pore during collapse and the subsequent $P V$ work involved in recompressing the vapor was responsible for the extreme heating that occurs in hot spots. Hatano [30] ${ }^{1}$ posited that

\footnotetext{
We think that the word "transverse" that appears in the final summary paragraph is a typographical error and the word "longitudinal" was intended.
} 
temperature alone is not sufficient to quantify the amount of chemistry occurring in simulations of pore collapse and supplemented temperature profiles with a measure of the number of energetic collisions in the pore (normalized by initial pore volume). He performed simulations on 3-D Lennard-Jones systems containing cuboid-shaped pores of varying size and found that increased cross-sectional area normal to the shock direction increases the number of energetic intermolecular collisions, whereas longer pore length parallel to the shock direction increases the peak temperature resulting from the collapse. Additionally, MD simulations have been used to study square pore collapse that produces jetting [31], the effect of shock strength on the mechanism of collapse [32], and the effect of pore size and arrangement on the minimum piston velocity necessary to produce detonation [33]. Reactive cases have been considered in MD simulations with the ReaxFF reactive force field $[34,35]$ for the energetic materials pentaerythritol tetranitrate (PETN) [36] and ammonium nitrate/fuel oil (ANFO) [37]. In both cases it was observed that the presence of pores led to an enhanced chemical reactivity compared to defect-free material.

The energetic material 1,3,5-trinitroperhydro-1,3,5-triazine (RDX, see Fig. 1a) has been widely studied both experimentally [38-45] and theoretically [35, 46-52]. There are four known polymorphs of RDX, denoted $\alpha$ [53], $\beta$ [54], $\varepsilon$ [55], and $\gamma$ [56]. The $\alpha$-phase is the stable form at room temperature and atmospheric pressure and has received the most study. Hooks and co-workers examined $\alpha$-RDX (hereafter, RDX) shock response using impact experiments [38], identified deformation mechanisms using nanoindentation [39], and studied decomposition using time-resolved emission spectroscopy [40]. Munday et al. [57] calculated generalized stacking fault energy surfaces using MD simulations to identify likely cleavage planes and slip planes to predict whether a given slip system will be brittle or ductile. Results obtained for the (010)[100], (021)[100], and (011)[100] slip planes were in good agreement with experiment. For the (010)[001] system, the authors predicted brittle response whereas experiments [39, 58] indicate this is a slip system. Theoretical calculations of Peierls stresses from atomic simulations to rank activated slip systems in RDX show agreement with the anisotropic plastic deformation response observed in experiment [59]. Strachan et al. [35] employed the ReaxFF [34] reactive force field to perform computer simulations on the thermal decomposition of RDX crystals. They observed an Arrhenius temperature dependence of the calculated characteristic reaction time.

Sewell and co-workers [41, 47-49] used MD simulations to identify and characterize plastic deformation mechanisms in single crystals of RDX subjected to shock waves propagating normal to the (100), (111), and (021) crystal planes. Cawkwell et al. [47] reported the formation of nano-scale shear bands for shocks propagating normal to (100). Cawkwell et al. [49] performed MD simulations in an attempt to explain an anomalous elastic-plastic response in the measured shock profiles for (111)-oriented RDX reported by Hooks et al. [38], and determined that this response resulted from a hardening effect created by the homogeneous nucleation of partial dislocation loops in the crystal at a well-defined shock strength. Ramos et al. [41] predicted using MD and verified experimentally using plate-impact experiments that a similar anomalous response would be obtained for shocks in (021)-oriented RDX due to commonalities in the slip systems activated by shocks on the (111) and (021) crystal planes. All of the shocks studied by Sewell and co-workers [41, 47-49] were performed using a non-reactive force field at low impact strengths; Rankine-Hugoniot shock pressures $P_{R H}$ ranging from 1.34 to $9.7 \mathrm{GPa}$, similar to those that might exist in accident scenarios. Nomura et al. [52] examined stronger impact strengths $\left(U_{P}=1.0\right.$ and $\left.3.0 \mathrm{~km} \mathrm{~s}^{-1}\right)$ using MD simulations with the ReaxFF force field to study shock propagation in (100)-oriented RDX that contained an initially $8 \mathrm{~nm}$ diameter spherical pore. They reported shockinduced vibrational excitation leading to bond breakage and the formation of $\mathrm{NO}_{2}, \mathrm{~N}_{2}$, and $\mathrm{H}_{2} \mathrm{O}$ in the vicinity of the pore collapse. They also reported an impact-strengthdependent pinning/de-pinning effect on the shock front due to passage over the pore surface and subsequent collapse.

In the present study we performed MD simulations of pore collapse in (100)-oriented $\alpha$-RDX for the case of shock wave passage over an initially $35.0 \mathrm{~nm}$ diameter cylindrical pore with the cylinder axis aligned parallel to the [010] crystal direction in the orthorhombic crystal (see Fig. 1). Impact velocities $U_{p}=1.0,2.0$, and $3.0 \mathrm{~km} \mathrm{~s}^{-1}$, corresponding to nominal shock pressures $P_{\mathrm{s}}=9.71$, 24.00 , and $42.48 \mathrm{GPa}$ in defect-free crystal, were considered as representative of weak, moderate, and strong shocks. Our main interest is in characterizing the nature of material flow, the nature and extent of energy and pressure localization, and molecular-scale disorder induced by the shock. The (100) impact plane was chosen for study because it produces a shock wave that propagates along a representative high-symmetry direction in the crystal, namely [100]. The $35.0 \mathrm{~nm}$ pore diameter was chosen because it was at the limit of what we could practically study without introducing strong artifacts in the results due to wave reflections at the simulation boundaries and the piston. The force field used for this work is non-reactive. Neglect of chemistry is reasonable at the lower shock pressures but is a gross approximation for the strong shock. Nevertheless, the qualitative information on material flow and energy localization as a function of impact strength is still a useful contribution to the knowledge base for pore 
Fig. 1 a An RDX molecule. Carbon is cyan, nitrogen is dark blue, oxygen is red, and hydrogen is black. Atoms N1 and $\mathrm{C} 4$ are used to define the vector discussed in "Rotational Order During Collapse" section. b Snapshot of the molecular center-of-mass locations in the simulation cell prior to shock. The colored regions around the pore surface and inside the black square define sets of molecules for which properties are monitored during collapse. In the chosen frame of reference the red, green, and purple arrows define angles of $45^{\circ}, 90^{\circ}$, and $135^{\circ}$, respectively, with the horizontal pore centerline that points toward the piston. The inset at the top right depicts concentric half-annuli around the pore surface that are also tracked as functions of time and provide a complementary view of the collapse. There is no relation between colors used in the main panel and those used in the inset. c Snapshot of the material after shock has been initiated but before the shock front has reached the pore (Color figure online)
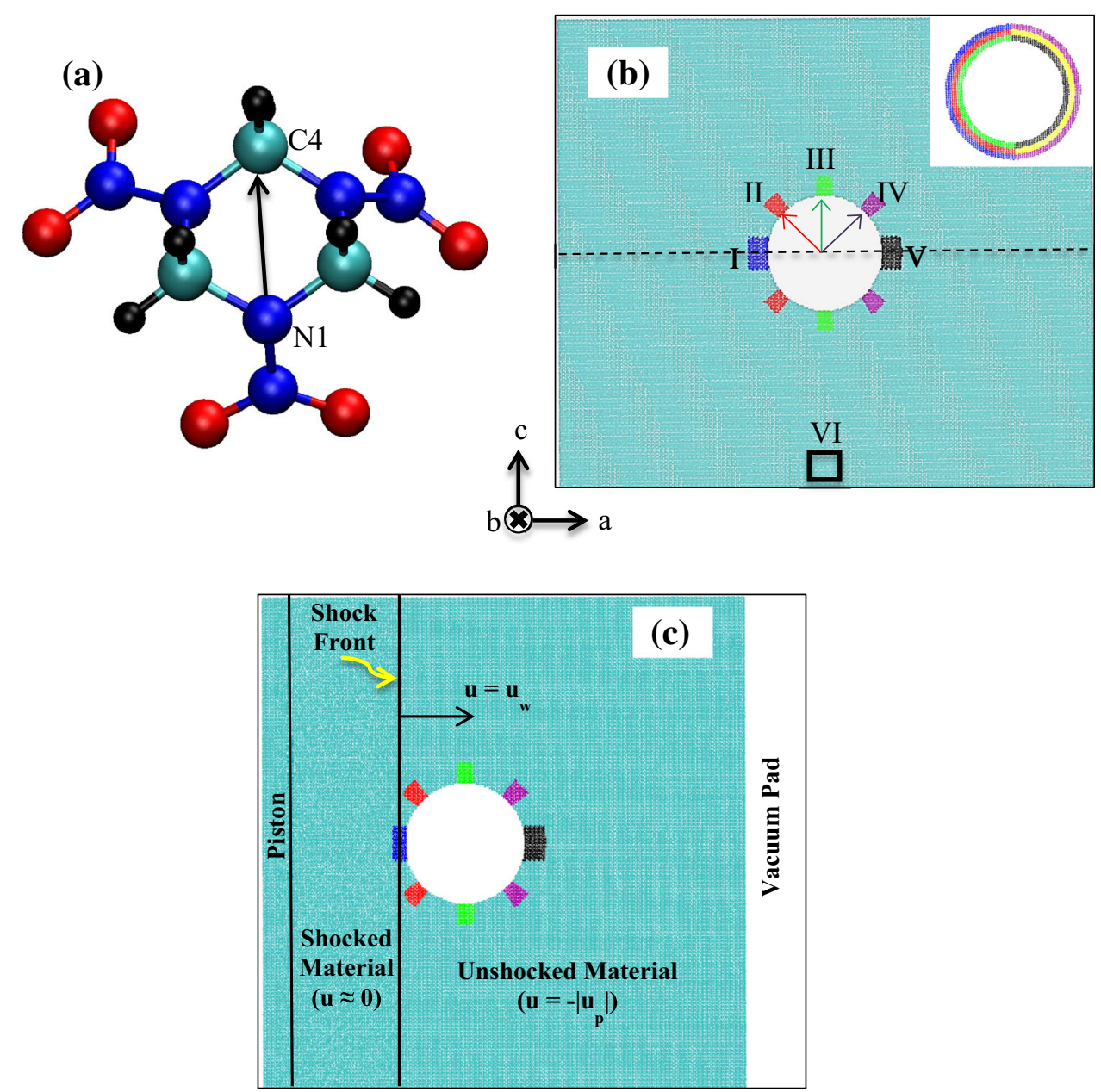

collapse in an anisotropic molecular crystal. The remainder of the manuscript is organized as follows: The force field and simulation details are provided in "Methods" section. Analysis, results, and discussion are presented in "Results and Discussion" section. Concluding remarks are given in "Conclusions" section.

\section{Methods}

The method of molecular dynamics is widely used to study fundamental properties and processes with atomic resolution. In MD, the classical equations of motion (i.e., Newton's equations or equivalently Hamilton's equations) are solved numerically for a system of atoms starting from a prescribed set of initial conditions (i.e., initial positions and momenta). This results in a phase space trajectory, typically of Cartesian atomic positions and momenta, from which any classical observable can be calculated. The equations of motion, initial conditions, and boundary conditions can be designed to simulate various statistical mechanical ensembles and physical scenarios ranging from isolated molecules or molecular collisions to chemically reacting condensed phases. A non-exhaustive listing of fundamental properties and phenomena that can be studied include energy transfer within and between molecules, thermal and mechanical properties, transport phenomena, deformation properties and mechanisms (dislocations, shear banding, etc.), phase transitions and chemistry, and connections between MD and continuum mechanics. MD methods are described in several texts, for example Refs. [60-62]. Applications of MD to energetic materials are discussed in relatively recent review articles [63, 64]. Arguably the most demanding aspect of an MD simulation is the need for accurate but computationally affordable interatomic forces. In most cases these are obtained from a parameterized analytical potential energy function although it is also possible in some cases to obtain the forces from quantum chemical (not quantum dynamical) calculations. Potential energy functions range in complexity from relatively simple "physical" force fields that accurately predict physical properties and processes but do not include the possibility of bond breaking or formation to more complicated "reactive" force fields such as ReaxFF 
$[34,35]$ and AIREBO $[65,66]$ that do treat chemistry. It is important to understand that the particles in MD are classical, thus inherently quantum mechanical effects such as zero-point energy and tunneling are not accounted for in MD simulations. In the present study we simulate the dynamics of shock waves using the microcanonical (i.e., constant energy) equations of motion with a simple parameterized physical force field that has been shown to accurately predict many fundamental thermal, mechanical, and transport properties of RDX.

All simulations were performed using the Largescale Atomic/Molecular Massively Parallel Simulator (LAMMPS) [67] code in conjunction with the fully flexible non-reactive nitramine potential energy surface (force field) by Smith and Bharadwaj (S-B) $[68,69]$. Three-dimensional periodic boundary conditions were used in all cases. In the S-B model the molecular topology is fixed, meaning chemical reactions cannot occur. The intramolecular potential energy is expressed as a sum over individual contributions from the covalent bonds (i.e., $\mathrm{C}-$ $\mathrm{N}, \mathrm{N}-\mathrm{N}, \mathrm{N}-\mathrm{O}$, and $\mathrm{C}-\mathrm{H}$ bonds), which are two-body terms, (see Fig. 1a); three-center angles (e.g., $\mathrm{C}-\mathrm{N}-\mathrm{C}$ or $\mathrm{O}-\mathrm{N}-\mathrm{O}$ ), which are covalent three-body terms involving the angle between two covalent bond vectors that share a common atom; and improper and proper dihedrals (e.g., $\mathrm{O}-\mathrm{N}-\mathrm{O}-\mathrm{N}$ and $\mathrm{O}-\mathrm{N}-\mathrm{N}-\mathrm{C}$, respectively), both of which are four-body terms. The non-bonded potential energy between atoms in different molecules, and between atoms in the same molecule but separated by three or more covalent bonds, is expressed as a sum of simple two-body pair potentials. Potentials for covalent bonds, three-center angles, and improper dihedrals are represented as harmonic functions. Dihedral potentials are represented by a sum of cosine terms. Non-bonded interactions are described by the Buckingham-plus-Coulomb potential with fixed atomic partial charges located on the nuclei. A $1.0 \mathrm{~nm}$ cutoff was used for the repulsion terms and the real part of the dispersion and electrostatic interactions. Long-range interactions were evaluated with the particle-particle particlemesh (PPPM) [70] algorithm with the relative RMS error in the forces set to $1 \times 10^{-4}$. While the S-B force field was originally developed for HMX, the methods by which it was formulated and parameterized were such that it should also be applicable to RDX; and Cawkwell et al. [47] and Munday et al. [51] determined that this is the case for both $\alpha$ - and $\gamma$-RDX. We obtained (300 K, $1 \mathrm{~atm})$ lattice parameters $a=1.3498 \mathrm{~nm}, \quad b=1.1554 \mathrm{~nm}$, and $c=$ $1.0564 \mathrm{~nm}$ based on simulations for a 3-D periodic $4 a \times 4 b \times 5 c$ supercell in the isostress-isothermal $(\mathrm{N} \sigma \mathrm{T})$ ensemble in which separate barostats were applied to each lattice direction and the cell overall was treated as triclinic. (The thermostat and barostat coupling parameters were set to 100.0 and $100.0 \mathrm{fs}$, respectively.) These lattice parameters compare well with the experimental values [53] $a=1.320 \mathrm{~nm}, \quad b=1.160 \mathrm{~nm}$, and $c=1.072 \mathrm{~nm}$; the values $a=1.3400 \mathrm{~nm}, b=1.1517 \mathrm{~nm}$, and $c=1.0623$ $\mathrm{nm}$ reported by Cawkwell et al. [47]; and $a=1.345 \mathrm{~nm}$, $b=1.153 \mathrm{~nm}$, and $c=1.053 \mathrm{~nm}$ reported by Munday et al. [51] for the same conditions. The calculated lattice parameters we obtained were used in the construction of all supercells described below.

Shock waves were generated in a reverse-ballistic configuration by impacting from the right with speed $U_{\mathrm{p}}$ a thermalized slab of flexible molecules (hereafter the flexible slab) onto a rigid, stationary piston composed of the same material (the piston). This results in a shock wave that propagates from left to right through the flexible slab with shock speed $U_{\mathrm{s}}=U_{\mathrm{w}}+U_{\mathrm{p}}$, where $U_{\mathrm{w}}$ is the speed of the wave measured in the frame of the stationary piston (see Fig. 1c). Impact speeds $U_{\mathrm{p}}=1.0,2.0$, and $3.0 \mathrm{~km} \mathrm{~s}^{-1}$ were studied. Corresponding shock pressures, calculated using the Rankine-Hugoniot relation $P_{\mathrm{s}}=\rho_{0} U_{\mathrm{s}} U_{\mathrm{p}}$, where $\rho_{0}$ is the pre-shock density of the crystal, were $P_{s}=9.71,24.00$, and $42.48 \mathrm{GPa}$, respectively. These pressures are within $1.94 \%$ of the longitudinal stress components $P_{\mathrm{xx}}$ calculated directly from the atomic stress tensor for defect-free crystal.

The supercell for the shock simulations was obtained by replicating the crystal unit cell by factors of 120,3 , and 135 along the $\hat{\boldsymbol{a}}, \hat{\boldsymbol{b}}$, and $\hat{\boldsymbol{c}}$ crystal directions, respectively, then adding a $20.0 \mathrm{~nm}$ vacuum pad parallel to the $\hat{\boldsymbol{a}}$ crystal direction. The purpose of the vacuum pad is to minimize interactions between the piston (which is created after equilibration just prior to initiation of the shock) and righthand side of the flexible slab across the periodic boundary along $\hat{\boldsymbol{a}}$. We aligned the $\hat{\boldsymbol{a}}$, or [100], crystal direction with the $x$-axis; the $\hat{\boldsymbol{b}}$, or [010], crystal direction with the $y$-axis; and the $\hat{\boldsymbol{c}}$, or [001], crystal direction with the $z$-axis. The size of the resulting slab of material, excluding the vacuum, was approximately $162.0 \mathrm{~nm} \times 3.5 \mathrm{~nm} \times 142.6 \mathrm{~nm}$ (see Fig. 1b). Justification for using a simulation cell that is thin along [010] comes from previous studies [47] where it was shown that, for shocks along [100], using a simulation cell that is thin along [010] does not lead to appreciable finitesize effects. A $35.0 \mathrm{~nm}$ diameter cylindrical pore with the cylinder axis aligned parallel to [010] was introduced by removing all molecules with center of mass within $17.5 \mathrm{~nm}$ of the midpoint of the slab in the $x$ and $z$ directions. Following the thermalization procedure described in the following paragraph, molecules in the first three unit cells $(\approx 4.0 \mathrm{~nm})$ from the left end of the simulation cell were defined as belonging to the piston and all others as belonging to the flexible slab. 
The simulation cell construction described in the preceding paragraph was used as the starting point for all three shock simulations. Different pseudorandom number seeds were used for each simulation. A 10.0 ps equilibration was performed in the isochoric-isoergic (NVE) ensemble using a 0.5 fs time step. During this equilibration instantaneous velocity re-scaling to yield total kinetic energy $E_{\mathrm{k}}$ corresponding to " $300 \mathrm{~K}$ " (based on $E_{\mathrm{k}}=3 N \kappa T / 2$, where $N$ is the number of atoms and $\kappa$ is the Boltzmann constant), was applied every $50.0 \mathrm{fs}$ and complete velocity re-selection from the $300 \mathrm{~K}$ Maxwell distribution was performed every $1.0 \mathrm{ps}$. The purpose of the velocity re-selection in addition to velocity scaling was to damp out the "breathing motion" along [100] that results from introducing the free surfaces. Next, 20.0 ps of Nosé-Hoover isochoric-isothermal (NVT) equilibration was performed using a $0.5 \mathrm{fs}$ time step with the thermostat coupling parameter set to 100.0 fs. Finally, starting from the phase space point at the end of the NVT equilibration, the shock wave was initiated by zeroing (and holding at zero) the velocities of atoms belonging to the piston and adding the impact velocity vector $\left(-U_{\mathrm{p}}, 0,0\right)$ to the instantaneous velocities of the atoms in the flexible slab. The shock simulations were performed in the NVE ensemble using a $0.2 \mathrm{fs}$ time step.

\section{Results and Discussion}

\section{Snapshots of Molecular Center-of-Mass Positions and Local Velocity Fields}

Molecular center-of-mass positions, recorded at $0.2 \mathrm{ps}$ intervals during the shock simulations, were used to monitor the progress of the shock. Snapshots of material in the vicinity of the pore at selected instants are shown for the $1.0 \mathrm{~km} \mathrm{~s}^{-1}$ shock in Fig. 2a, c, e, the $2.0 \mathrm{~km} \mathrm{~s}^{-1}$ shock in Fig. 3a, c, e, and the $3.0 \mathrm{~km} \mathrm{~s}^{-1}$ shock in Fig. $4 \mathrm{a}, \mathrm{c}$, e. For each shock strength the snapshots correspond to times: (a) during collapse approximately halfway to pore closure; (c) immediately after pore closure; and (e) at the time of maximum compression (that is, when the leading edge of the shockwave, which is curved immediately after passage over the pore, reaches the right-hand end of the sample). Only the upper halves of the simulation cells are shown due to symmetry about the equator of the pore. The colors in the snapshots correspond to those in Fig. 1b, which also serves as a reference for the positions of molecules prior to shock passage. The colored regions on the pore surface and inside the black square in the main panel of Fig. 1b designate sets of molecules that will be the focus of much of the analysis and are labeled as follows: the ejection point (blue, Set I); $\pm 45^{\circ}$ to the centerline of the pore (red, Set II); the upper and lower poles of the pore (green, Set III); $\pm 135^{\circ}$ to the centerline of the pore (purple, Set IV); the stagnation region or downstream wall (black, Set V); and bulk material away from the pore (Set VI). These regions were used to characterize the response of material as a function of initial position on the pore surface. The inset in the top-right corner of Fig. $1 \mathrm{~b}$ contains a second scheme for coloring the molecules that highlights different aspects of the collapse. The molecules in both representations retain their color-ID during the simulations and thus convey qualitative information about the nature of overall material deformation during collapse. There is no relationship between the colors used in the two representations. Where appropriate below, averaging over symmetryequivalent regions of material above and below the equator was performed to improve the statistics.

Local velocity fields, calculated at the same instants as shown in panels (a, c, e) of Figs. 2, 3, 4, are shown in panels $(b, d, f)$ of those figures and are used to convey instantaneous flow on the microscopic scale. The velocity fields were calculated in an Eulerian frame by dividing the simulation cell into a 3-D grid of smaller cells in the $(x-$ $z$ ) plane. The width and height for each cell are equal to the lattice spacing in the corresponding direction, that is, $1.3498 \mathrm{~nm}$ along the $x$-axis and $1.0564 \mathrm{~nm}$ along the $z$ axis, and the depth extends all the way through the sample in the $y$-direction. Entire molecules were assigned to the cells based on their instantaneous center-of-mass positions. The $x$ - and $z$-components of the center-of-mass velocity, $v_{x}$ and $v_{z}$, respectively, were calculated for the material in each cell at a given instant of time and plotted as a vector, with the origin of a given vector located at the geometric center of the corresponding cell.

Many of the experiments and simulations referenced in the Introduction exhibited a hydrodynamic response during pore collapse, characterized by the focusing of upstream wall material into a fluid-like jet directed along the centerline of the pore. The collapse resulting from the weakest shock we studied (1.0 $\mathrm{km} \mathrm{s}^{-1}$, Fig. 2) shows no signs of jet formation and is dominated by convergence of mainly upstream-side wall material toward the centerline and downstream wall (Fig. 2a, c, e). In terms of the sets of molecules identified in Fig. 1b, the collapse is led by material in Set II flowing into the pore at an angle of approximately $45^{\circ}$ to the horizontal. Material initially located in the ejection zone (Set I) initially lags behind but accelerates as the collapse progresses such that 'frontier' molecules in Sets I and II arrive at the downstream wall at approximately the same time. Although material in the vicinity of the upstream side of the pore shows signs of intense plastic deformation (i.e., translational disorder), note the persistence of regions of local crystallinity (i.e., relatively high translational order) in some of the material located between Sets I and II throughout the collapse. 

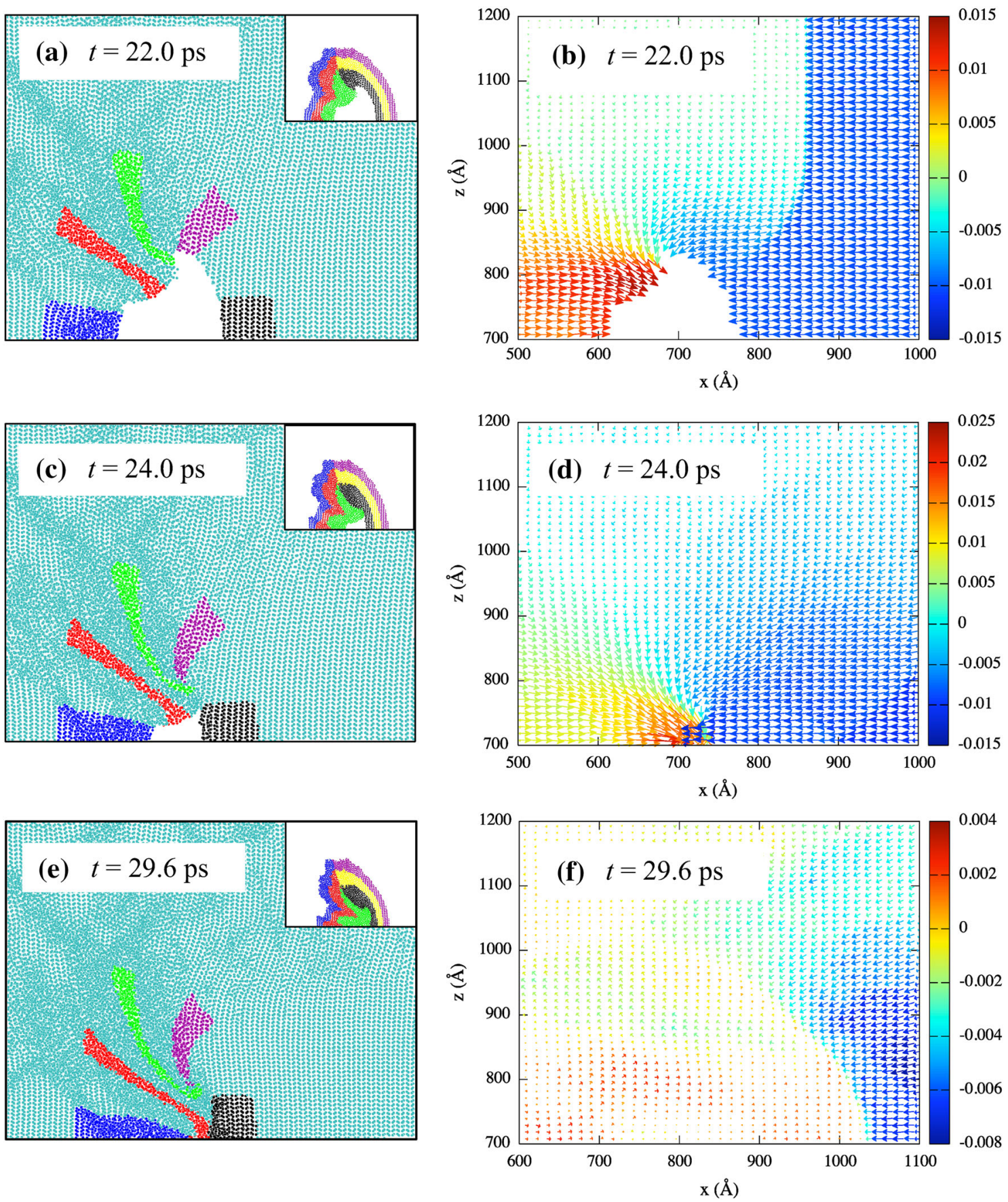

Fig. 2 Left panels a, c, and $\mathbf{e}$ Snapshots of molecular centers of mass during pore collapse at three instants of time for the $1.0 \mathrm{~km} \mathrm{~s}^{-1}$ shock. Colors correspond to those shown in Fig. 1b and illustrate the qualitative features of the mass flow during collapse. Right panels $\mathbf{b}$, $\mathbf{d}$, and $\mathbf{f}$ Velocity fields for the same instants of time as in the left

Velocities in the vicinity of the collapse zone (Fig. 2b, d, f) are close to zero confirming that net translational motion (local mass flow) has halted shortly after collapse. At the time of maximum compression the 'translational strain' in the defined sets of molecules initially located at the pore column. Units for the velocity vector are $\AA \mathrm{fs}^{-1}$. Times are relative to the instant when the shock is initiated. Only the upper half of the simulation is cell is shown and the view is restricted to material in the vicinity of the pore (Color figure online)

surface is greatest for Sets II and III (red and green, respectively) and least for Set $\mathrm{V}$ (black).

The moderate shock $\left(2.0 \mathrm{~km} \mathrm{~s}^{-1}\right.$, Fig. 3) results in stronger material focusing during collapse compared to the result obtained for the weak shock case. The molecular 

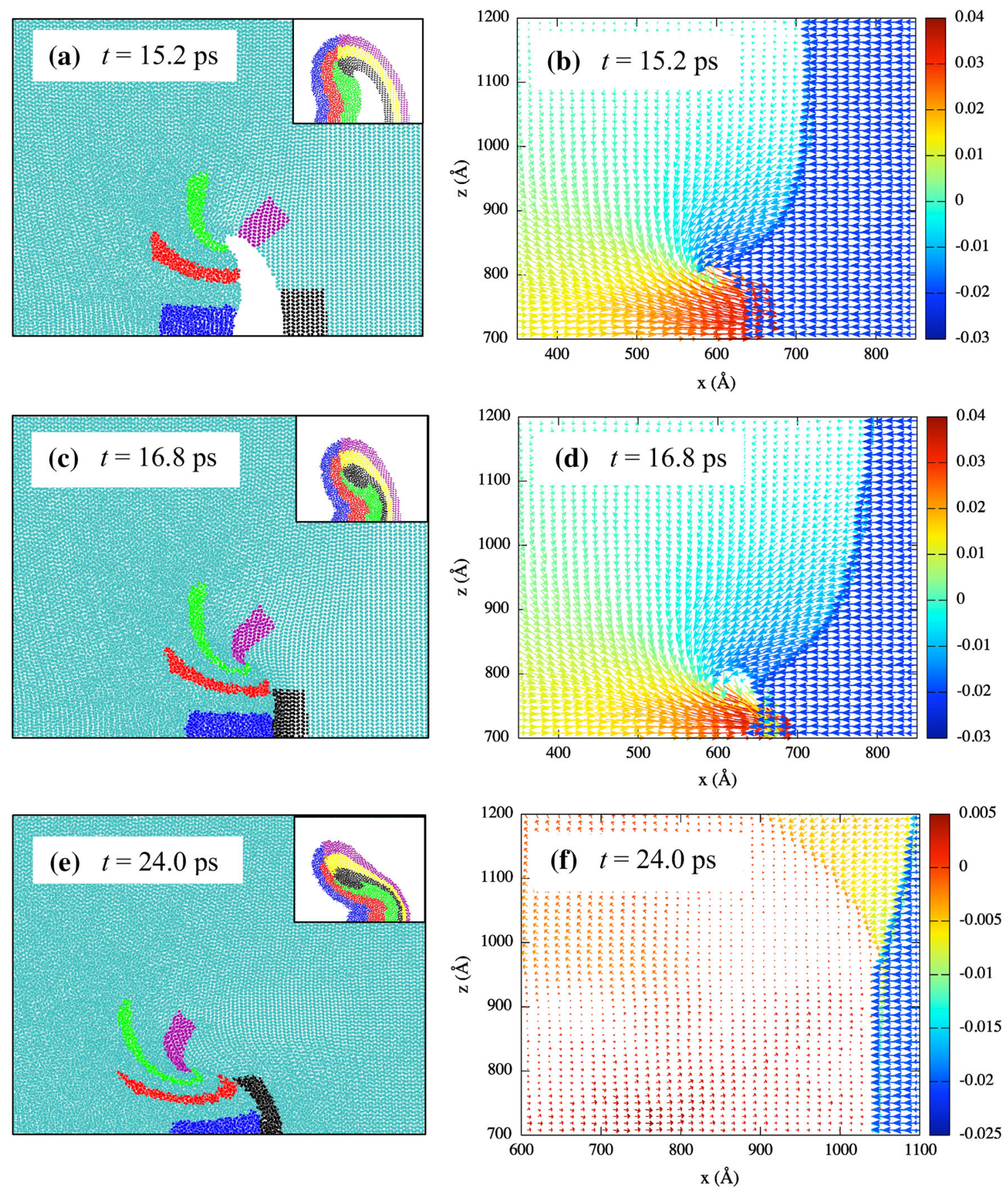

Fig. 3 As in Fig. 2, but for the $2.0 \mathrm{~km} \mathrm{~s}^{-1}$ impact (Color figure online)

velocities of the ejection zone (Set I) and Set II are more strongly oriented along a common axis parallel to the shock direction (Fig. 3b). The material initially in the pole region (Set III) flows toward the centerline with the upstream wall material, producing an enhanced focusing of material with a large component of motion parallel to the centerline. Regions of vorticity are evident in the velocity field during collapse (Fig. 3b and d) as material in Sets III and IV flow toward the centerline after entrainment with ejection zone material. The impact of the ejection zone material onto the downstream wall is much stronger than for the weak shock, resulting in more significant deformation of downstream wall material (Fig. 3e). A subsequent flow develops in the vicinity of the impact zone (Fig. 3d) that has stagnated by the time of maximum compression as evidenced by velocities that are near zero in Fig. 3f.

The strong shock (3.0 $\mathrm{km} \mathrm{s}^{-1}$, Fig. 4) exhibits an even stronger focusing of material along the pore centerline. 

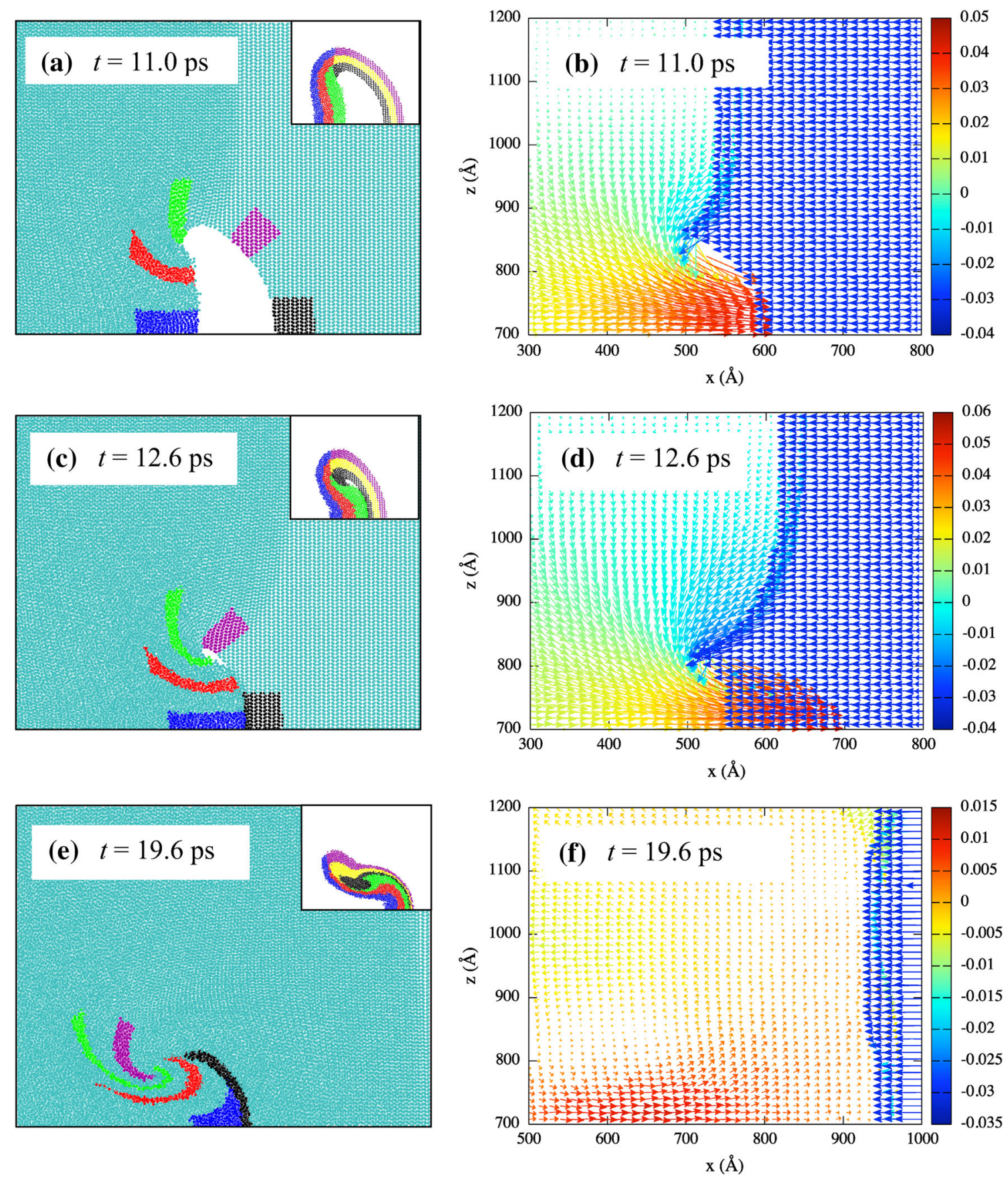

Fig. 4 As in Fig. 2, but for the $3.0 \mathrm{~km} \mathrm{~s}^{-1}$ impact (Color figure online)

Ejection of molecules in Set I with high velocities (Fig. 4b) in conjunction with convergence of the flow of most of the upstream wall material parallel to the horizontal centerline of the pore causes the near-pore material to involute and form a jet (Figs. 4a, c). Compared to the weaker shocks, impact produces a much more severe deformation of the downstream wall material that severely displaces molecules away from a well-ordered crystalline state (Fig. 4e). After pore closure, persistent following flow exists along with regions of vortex-like motion centered on the lobes that pinch shut to complete collapse (Fig. 4f).

As expected, snapshots and velocity fields suggest mechanisms of collapse that depend on the initial impact strength. Weak shock passage results in pore collapse via a visco-plastic flow of material that largely ceases when the pore pinches shut, with little penetration of material into the downstream region or residual vorticity in the inflow regions. The moderate shock results in more focusing of 
the upstream wall material with early signs of jet formation and some amount of penetration into the downstream wall. By contrast, the strong shock focuses material and velocities along the pore centerline strongly enough to create a hydrodynamic jet that penetrates significantly into the downstream wall along with considerable vorticity in the inflow region. The greater focusing of material with increasing impact strength should result in a greater deposition of energy at the stagnation zone, leading to the formation of an intense mechanical hot spot.

\section{Rotational Order During Collapse}

Time autocorrelation functions [71] for a time-dependent vector, defined individually in the lab frame for each molecule, were calculated for the molecules in Sets I-V to quantify the extent of molecular rotational motion induced by pore collapse as a function of initial location at the pore surface, and for molecules in Set VI as a point of reference for molecules far from the pore. To calculate the autocorrelation functions, for each molecule $m$ in a given set the unit vector $\hat{\boldsymbol{r}}(t)$ that points from the ring nitrogen atom N1 to carbon atom $\mathrm{C} 4$ (see Fig. 1a) was monitored as a function of time $t$. The time autocorrelation function (ACF) of $\hat{\boldsymbol{r}}$ for molecule $m$ was calculated as

$C_{m, \hat{r}}(\tau)=\frac{T}{T-\tau} \sum_{\tau=0}^{T-\Delta t} \sum_{t^{\prime}=0}^{T-\tau-\Delta t} \frac{\hat{r}\left(t^{\prime}\right) \hat{r}\left(t^{\prime}+\tau\right)}{\hat{r}\left(t^{\prime}\right) \hat{r}\left(t^{\prime}\right)}$,

where $t^{\prime}$ is a particular time origin, $\tau$ is the positive time lag from that time origin, $T$ is the total interval of time observed ( 2.0 ps per time window, as elaborated below), and $\Delta t$ is the spacing between successive data points. All time origins $t^{\prime}$ within a given time window are treated as equivalent. The range of $C_{m, \hat{r}}(\tau)$ is $[-1,+1]$ and for a liquid the long-time value should approach zero. The ACF $C_{R, \hat{r}}(\tau)$ for the molecules in a given set (I-VI) was defined as the arithmetic average of $C_{m, \hat{r}}(\tau)$ over all $M$ molecules assigned to that set:

$C_{R, \hat{r}}(\tau)=\frac{1}{M} \sum_{m=1}^{M} C_{m, \hat{r}}(\tau)$

The analysis was performed separately for contiguous $2.0 \mathrm{ps}$ time windows starting at the instant when the shock wave reached the upstream pore wall and continuing until the time of maximum compression. The results are presented in Fig. 5, where the dashed vertical lines denote the approximate times of pore closure. Additionally, the ACFs presented in Fig. 5 were fit to the exponential function,

$C(\tau)=A e^{-\frac{\tau}{\alpha}}$,

to obtain the characteristic rotational time constant $\alpha$ that defines an approximate time scale on which, on average,
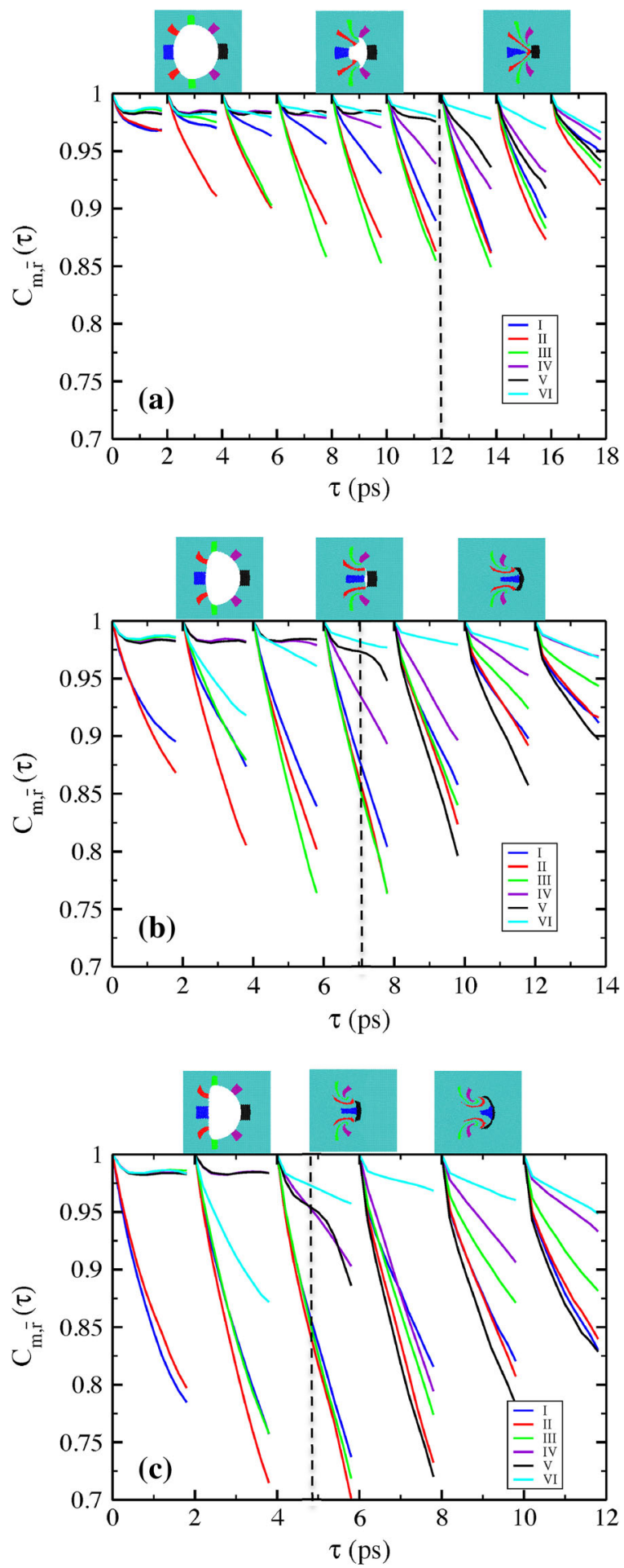

Fig. 5 Rotational autocorrelation functions during and after pore collapse. a $U_{p}=1.0 \mathrm{~km} \mathrm{~s}^{-1}$, b $U_{p}=2.0 \mathrm{~km} \mathrm{~s}^{-1}$, and $\mathbf{c} U_{p}=3.0 \mathrm{~km} \mathrm{~s}^{-1}$. $\mathrm{ACFs}$ are calculated in contiguous $2.0 \mathrm{ps}$ time windows starting when the shock reaches the upstream pore wall. The time windows are offset horizontally in the figures for ease of viewing. Line colors coincide with previously defined sets. The vertical dashed lines indicate the approximate times of pore closure. Insets are placed above the curves as representative snapshots of the simulations around the times of analysis (Color figure online) 
the molecules in a particular region lose memory of their initial orientation within a given $2.0 \mathrm{ps}$ time window. Smaller $\alpha$ corresponds to faster rotational disordering. Values of $\alpha$ are presented in Table 1 for four selected $2.0 \mathrm{ps}$ time windows for each shock strength. These time constants are not intended as rigorous metrics for rotational diffusion in RDX but instead provide a consistent method for comparing the different extents of rotational motion at different locations and time windows during collapse for the different impact strengths.

In the results from "Snapshots of Molecular Center-ofMass Positions and Local Velocity Fields" section it was seen that passage of the weak shock wave $\left(1.0 \mathrm{~km} \mathrm{~s}^{-1}\right.$ impact) collapses the pore in a visco-plastic manner characterized by convergence of mainly upstream wall material along the centerline. The molecular-scale rotational motion as characterized by the autocorrelation function during collapse (Fig. 5a) is consistent with this observation. Molecules in Sets II and III along the upstream wall (red and green curves, respectively) are ejected into the pore at about a $145^{\circ} \mathrm{I}$ angle to the direction of shock propagation (see Fig. 2a and b) producing strong molecular torques that cause increased rotational motion of the molecules in these sets. By contrast, ejection zone material (Set I, blue curve) is ejected into the pore essentially parallel to the shock direction producing less torque on average on the molecules at early times so that less rotational motion occurs compared to the molecules in Sets II and III. These observations are supported by the rotational time constants (see Table 1a); Sets II and III exhibit much smaller time constants ( $\approx 18$ ps) than Set I ( $\approx 56$ ps) in the early stages of collapse (4-6 ps time window). It is not until the late stages of collapse (10-12 ps time window), when molecules initially surrounding the pore are all converging to the "pinch point," that the time constant for Set I approaches those for Sets II and III. Throughout the collapse, Sets II and III exhibit more rotational motion (lower values of $C_{R, \hat{r}}(\tau)$ at a given $\tau$ ) than the ejection zone material and the ACFs for those three sets exhibit greater decay in all time windows than do sets initially on the downstream wall (Sets IV-V) or far from the shock (Set VI).

For the moderate and strong shocks, 2.0 and $3.0 \mathrm{~km} \mathrm{~s}^{-1}$, Fig. $5 \mathrm{~b}$ and $\mathrm{c}$ and Table $1 \mathrm{~b}$ and $\mathrm{c}$, respectively, the ACFs for Sets II and III still exhibit the most rapid and greatest magnitude decay during approximately the first half of collapse, but rotational motion in Set I is now similar to these other upstream wall regions (Fig. $5 \mathrm{~b}$ and c, 0-2 ps and 2-4 ps time windows). Indeed, for the stronger shocks, up until pore closure the rotational time constants for Sets I, II, and III are all within $\approx 6$ ps of each other for the $2.0 \mathrm{~km} \mathrm{~s}^{-1}$ shock (Table 1b, 2-4 ps time window) and within less than 2 ps for the $3.0 \mathrm{~km} \mathrm{~s}^{-1}$ shock (Table 1c, 2-4 ps time window).
Table 1 Rotational time constants for RDX

\begin{tabular}{|c|c|c|c|c|}
\hline \multirow[t]{2}{*}{ Set } & \multicolumn{4}{|c|}{ Time window (ps) } \\
\hline & $4-6$ & $10-12$ & $12-14$ & $16-18$ \\
\hline \multicolumn{5}{|c|}{ (a) $U_{p}=1.0 \mathrm{~km} \mathrm{~s}^{-1}$} \\
\hline I & 55.6 & 15.6 & 12.8 & 40.0 \\
\hline II & 17.9 & 12.7 & 12.7 & 25.0 \\
\hline III & 18.2 & 11.8 & 11.4 & 31.2 \\
\hline IV & - & 31.2 & 22.2 & 50.0 \\
\hline V & - & - & 30.3 & 33.3 \\
\hline VI & - & - & - & 62.5 \\
\hline \multirow[t]{2}{*}{ Set } & \multicolumn{4}{|c|}{ Time window (ps) } \\
\hline & $2-4$ & $6-8$ & $8-10$ & $12-14$ \\
\hline \multicolumn{5}{|c|}{ (b) $U_{p}=2.0 \mathrm{~km} \mathrm{~s}^{-1}$} \\
\hline I & 14.5 & 8.6 & 12.8 & 22.7 \\
\hline II & 8.3 & 7.0 & 9.9 & 23.3 \\
\hline III & 14.1 & 6.9 & 10.8 & 37.0 \\
\hline IV & - & 16.7 & 17.0 & 66.7 \\
\hline $\mathrm{V}$ & - & 45.4 & 8.4 & 18.5 \\
\hline VI & 21.7 & - & - & 66.7 \\
\hline \multirow[t]{2}{*}{ Set } & \multicolumn{4}{|c|}{ Time window (ps) } \\
\hline & $2-4$ & $4-6$ & $6-8$ & $10-12$ \\
\hline \multicolumn{5}{|c|}{ (c) $U_{p}=3.0 \mathrm{~km} \mathrm{~s}^{-1}$} \\
\hline I & 6.7 & 6.1 & 9.6 & 10.8 \\
\hline II & 5.4 & 5.4 & 6.0 & 11.5 \\
\hline III & 6.5 & 5.6 & 7.4 & 15.9 \\
\hline IV & - & 18.9 & 7.9 & 30.3 \\
\hline $\mathrm{V}$ & - & 17.9 & 5.7 & 10.6 \\
\hline VI & 13.5 & - & - & 40 \\
\hline
\end{tabular}

Units are ps. Time windows coincide with those presented in Fig. 5. Dashes in the table indicate curves in Fig. 5 that are not well described by an exponential decay

Results to the right of the vertical dashed lines in Fig. 5 are for times between initial pore closure and maximum compression. From the simulation snapshots and velocity fields shown in Figs. 2, 3, 4 it is clear that material ejected from the upstream wall stagnates quickly upon impacting the downstream wall for the weak shock, with little further mass flow once the pore has closed; whereas increasing amounts of post-closure flow (penetration) of the upstream wall material into the downstream wall occurs for the moderate and strong shocks. The material stagnation in the vicinity of the collapse zone for the weak shock case is apparent in the autocorrelation function (Fig. 5a). Immediately following collapse (12-14 ps time window), the collapse zone material (Sets I-V) does not rotationally disorder to a much greater degree than material far from the pore (Set VI) that has mainly only been uniaxially 
compressed and heated by the shock. By the time of maximum compression (16-18 ps time window), rotational motion has mostly stagnated throughout the sample.

The collapse-zone regions (Sets I-V) in the moderate shock case (Fig. 5b; Table 1b) exhibit more rotational motion after collapse than for the weak shock. It was observed in connection with Fig. 3 that although there is some degree of focusing for the moderate shock case, the focusing is not strong enough to result in a well-formed jet. While there is some penetration into the downstream wall, rotational motion has largely ceased by the time of maximum compression similar to the weak shock (Fig. 5b, 12-14 ps time window). It should also be noted that the temperature and pressure are higher in the vicinity of the collapse zone compared to material away from the pore (see "Temperature and Pressure" section). The higher temperature (higher kinetic energy) in conjunction with structure disruption due to penetration of the downstream wall will favor increased rotational motion. However, the higher pressure from compression will serve to reduce the amount of rotational motion. These competing factors probably explain why the results far from the pore (Set VI) are almost quantitatively similar at long times for the two weaker shocks.

Increased rotational motion after pore closure is observed for the strong shock case compared to the two weaker shocks (see Fig. 5c; Table 1c). The rotational time constants for collapse zone material (Sets I-III) immediately after collapse for the 1.0 and $2.0 \mathrm{~km} \mathrm{~s}^{-1}$ shocks are in the $10-13 \mathrm{ps}$ range whereas for the $3.0 \mathrm{~km} \mathrm{~s}^{-1}$ shock smaller time constants in the 6-10 ps range are obtained. There is also evidence in the autocorrelation function for the strong shock of considerable rotational motion at the time of maximum compression (Fig. 5c; Table 1c 10-12 ps time window). This result is the combined effect of the heating, jet penetration, and persistent high-strain mass flow in the strong shock case.

\section{Temperature and Pressure}

Intramolecular (i.e., rotational-vibrational) temperatures and pressures were calculated as functions of location in the sample for several instants of time during pore collapse using the same Eulerian grid described in "Snapshots of Molecular Center-of-Mass Positions and Local Velocity Fields" section. The intramolecular temperatures, hereafter temperatures, were calculated using a previously described method [72]. The atomic stress tensor for all atoms was saved at $0.2 \mathrm{ps}$ intervals during the simulation, and pressures were then calculated using $P=-\left(\sigma_{x x}+\sigma_{y y}+\sigma_{z z}\right) / 3$, for all atoms in a given Eulerian grid cell at a given time. Figure 6 contains color maps of temperature for all three shock pressures at two times of interest: immediately after initial pore closure (left-hand column) and at the time of maximum compression (right-hand column). Results for the weak, intermediate, and strong shock are shown in the upper, middle, and lower rows (panels (a) and (b), (c) and (d), and (e) and (f), respectively). Figure 7 contains profiles of temperature (left-hand column) and pressure $P$ (righthand column) along the shock direction, for material within $5.0 \mathrm{~nm}$ of the horizontal centerline, at four different instants after the collapse: when the pore first closes (black solid curves), the time of maximum compression (blue solid curves), and two intermediate times (red and green dashed curves). Figure 6 provides a qualitative overall description of the temperature distribution in the material whereas Fig. 7 provides a more quantitative measure of the peak temperature and pressure achieved and how those quantities evolve along the centerline as the collapse occurs.

The temperature maps for the weak shock just after pore closure and at the time of maximum compression are shown in Fig. 6a and b, respectively, and the corresponding temperature and pressure profiles along the centerline at those times are shown as black and blue

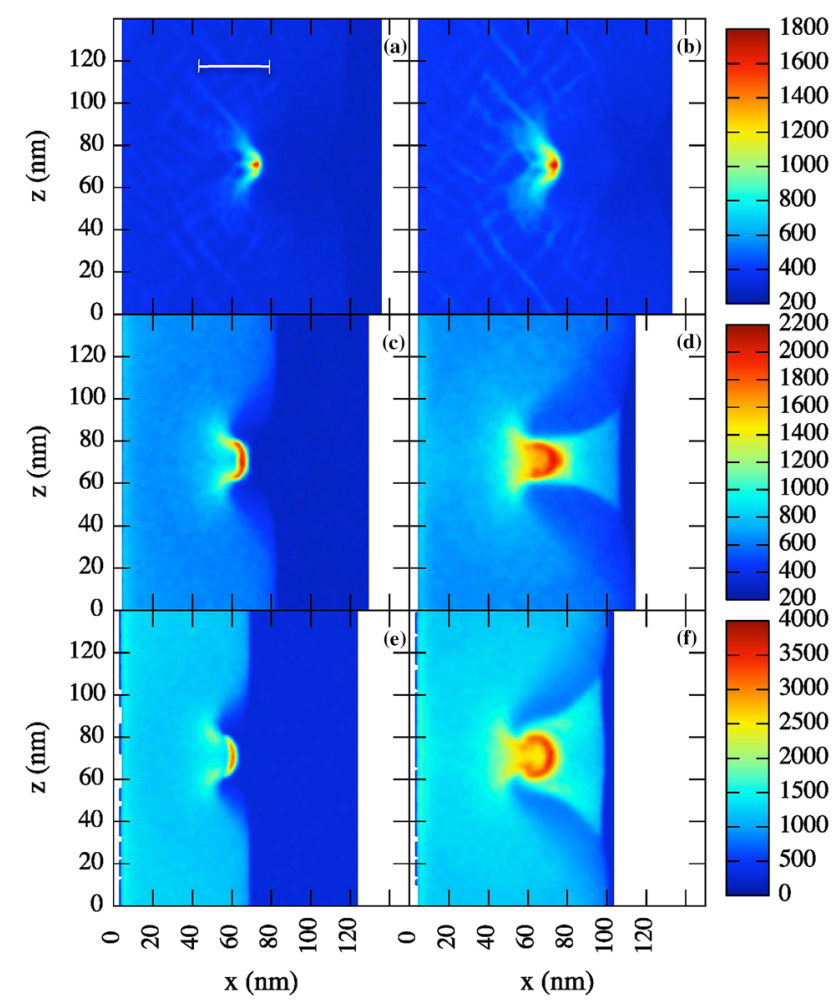

Fig. 6 Rotational-vibrational temperatures just after pore closure (left column, panels a, c, and e) and at the time of maximum compression (right column panels $\mathbf{b}, \mathbf{d}$, and f) for $U_{p}=1.0 \mathrm{~km} \mathrm{~s}^{-1}$ (top row), $2.0 \mathrm{~km} \mathrm{~s}^{-1}$ (middle row), and $3.0 \mathrm{~km} \mathrm{~s}^{-1}$ (bottom row). Note that the temperature scale differs for each impact strength. The white line in panel a depicts the initial pore diameter, $35.0 \mathrm{~nm}$, and is intended merely as a visual aid (Color figure online) 
solid curves, respectively, in Fig. 7a and b. Peak temperatures of $\approx 1500 \mathrm{~K}$ and pressures of $\approx 20 \mathrm{GPa}$ are achieved in the vicinity of the collapse zone within about 1 ps of closure (dashed red curves); corresponding temperature and pressure values away from the pore are $\approx 400 \mathrm{~K}$ and approximately $\approx 9.8 \mathrm{GPa}$. From the temperature map, heating is limited to a relatively small area in the vicinity of the stagnation point (see Fig. 6a and b); and after modest heating during the first ps after closure,
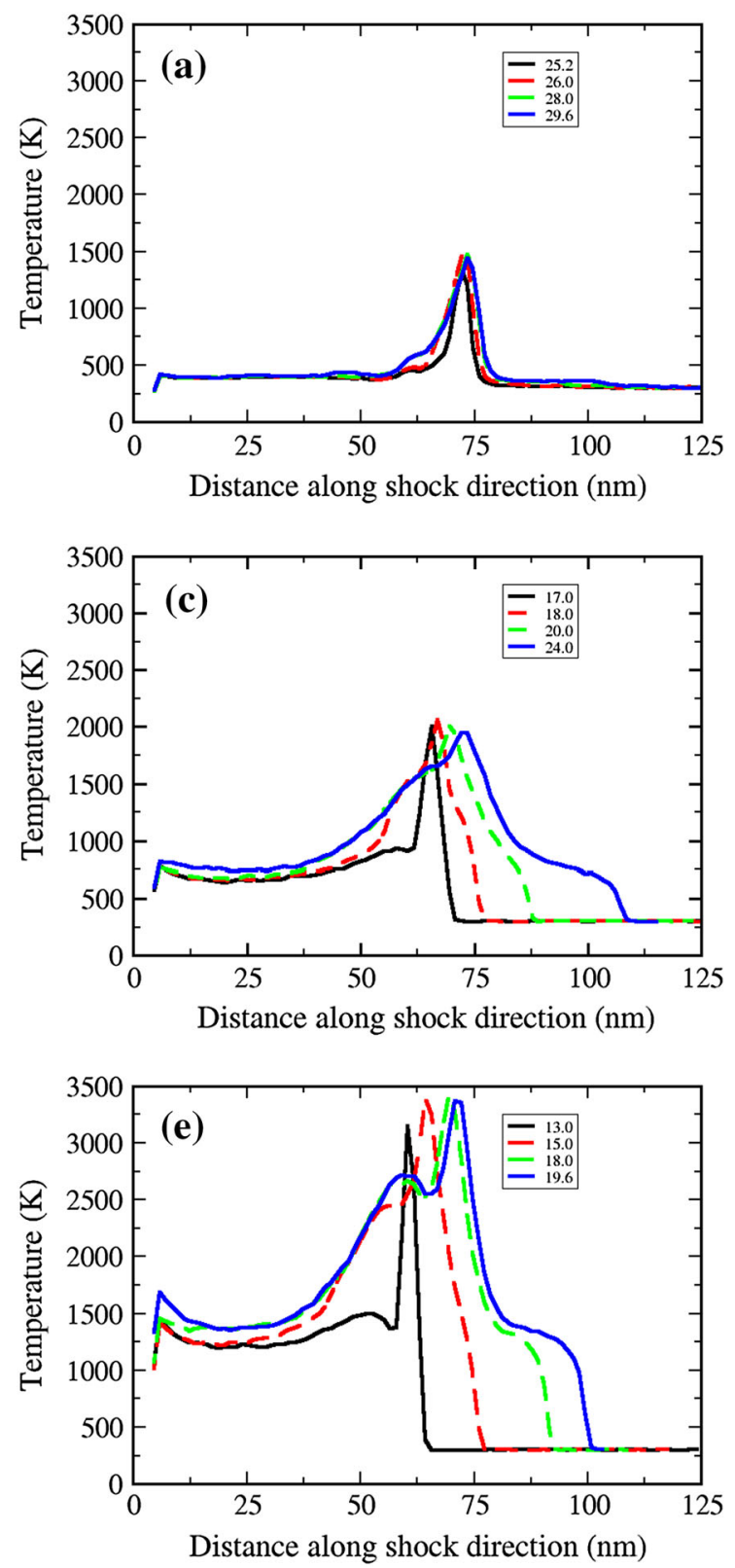

Fig. 7 Spatial profiles of temperature (left column) and pressure (right column, only pressures behind the shock are shown) parallel to the shock direction. The results are for a strip of material located within $5 \mathrm{~nm}$ of the horizontal centerline of the simulation cell for three different impact speeds $U_{p}$. Panels a, b $U_{p}=1.0 \mathrm{~km} \mathrm{~s}^{-1}$; the temperature profile remains relatively constant in the time that elapses between initial pore closure and maximum compression of the system $4.4 \mathrm{ps}$ later (see Fig. 7a).

In contrast to the temperature profiles for the weak shock, the pressure profiles (see Fig. $7 \mathrm{~b}$ ) broaden and decrease in amplitude quickly after pore closure. At the time of initial closure (25.2 ps, black solid curve) there is a narrow pressure spike. Pressure waves reflecting from the
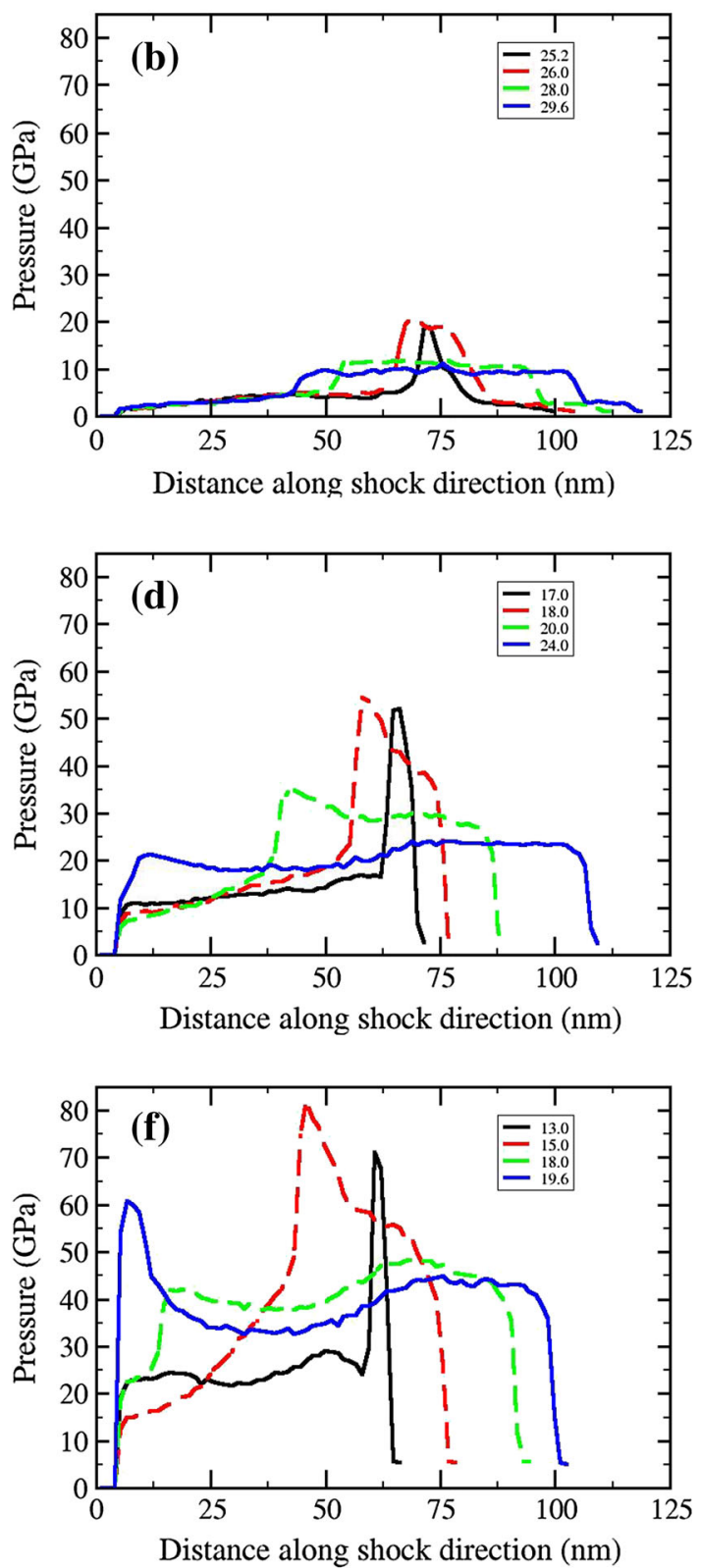

panels c, d $2.0 \mathrm{~km} \mathrm{~s}^{-1}$; panels e, f $3.0 \mathrm{~km} \mathrm{~s}^{-1}$. Lines are colored according to elapsed time, in ps, since initiation of the shock, with the shortest (black) and longest (blue) elapsed times depicted using solid curves and the intermediate times (red and green) depicted using dashed curves (Color figure online) 
stagnation point increase the pressure slightly (red dashed curve), after which the two waves quickly decrease in amplitude as they move forward and backward through the material. The $10 \mathrm{GPa}$ peak pressure at the time of maximum compression (blue solid curve value at $\approx 110 \mathrm{~nm}$ ) is close to the pressure of $9.71 \mathrm{GPa}$ expected from shock compression in the defect-free crystal of RDX.

Pore collapse resulting from the moderate shock has a greater effect on heating material surrounding the collapse zone than for the weak shock case. Once again a localized region of higher temperatures is visible just after collapse (Fig. 6c). However, in contrast to the result discussed above for the weak shock, in this case temperature at maximum compression (Fig. 6d) exhibits a profile that has evolved considerably since initial closure. The area of the hotter material has increased from Fig. 6c to d and a "fan" is visible between the shock wave and the collapse zone in Fig. 6d. The peak temperature along the centerline is $\approx 2000 \mathrm{~K}$ (Fig. 7c) and remains fairly constant in time. However, in contrast to the weak shock case, here the peak broadens considerably over that same time interval. The causes for this are a combination of convection and redistribution of energy from the molecular translational degrees of freedom in the flow to intramolecular kinetic energy in the stagnated region.

A secondary compressive wave centered on the collapse zone is visible in the pressure profiles (Fig. 7d). Similar to the $1.0 \mathrm{~km} \mathrm{~s}^{-1}$ impact case, pressure waves that quickly decrease in amplitude are reflected from the stagnation point. The peak pressure value in Fig. $7 \mathrm{~d}$ at the time of maximum compression is $\approx 24 \mathrm{GPa}$, which is the value predicted by the Rankine-Hugoniot shock pressure of $24.00 \mathrm{GPa}$, but much less than the peak pressure, $\approx 50$ $\mathrm{GPa}$, at the time of initial pore closure 7 ps earlier.

The evolution of the temperature distribution becomes much more pronounced when considering the strong shock wave generated by the $3.0 \mathrm{~km} \mathrm{~s}^{-1}$ impact (Fig. 6e and f). Similar to the moderate shock case, an arc of localized heating is visible just after collapse (Fig. 6e) corresponding to recompression and partial stagnation of molecules against the downstream pore wall. Additionally, there are two lobes of heated material located just above and below the centerline and $\approx 10 \mathrm{~nm}$ behind the leading edge of the stagnation region. These lobes coincide with the small vortex regions generated near the end of pore closure (Fig. 4d). The temperature distribution changes noticeably in the time interval between cavity closure and maximum compression, with the collapse zone material convecting heat to surrounding material. Once again, the temperature peak broadens in the time interval between closure and maximum compression (Fig. 7e). An initial narrow peak at about $3100 \mathrm{~K}$, just after closure at $13 \mathrm{ps}$, is visible that broadens considerably with increasing time after closure.
The increased peak broadening is attributed to increased amounts of convection due to the greater penetration of the downstream wall and more translational kinetic energy from the stronger shock that is redistributed into intramolecular energy.

As for the intermediate shock strength, a secondary compression wave is evident in the pressure profiles for the strong shock case (Fig. 7f). An initial pressure spike to $\approx 73 \mathrm{GPa}$ is visible shortly after closure at $13 \mathrm{ps}$. Reflected waves originating at the stagnation point travel out through the material recompressing the system. Once again, the amplitude of the reflected waves decreases quickly as they travel through the system. The peak pressure at maximum compression is $\approx 43 \mathrm{GPa}$, which compares to the calculated Rankine-Hugoniot shock pressure of $42.48 \mathrm{GPa}$. (The intense peak located near the piston at $19.6 \mathrm{ps}$ is taken to be an artifact of the fast traveling wave reflecting off of the piston.)

Ideally one would like to monitor the samples for longer times to determine whether or to what extent pore collapse affects the long-time structural properties. However, the time scale available in the current studies is limited by the shock wave reaching the right-hand side of the sample. Shock front absorbing boundary conditions have been employed in previous studies $[47,73]$ to extend the amount of simulation time available for data analysis. However, the SFABC method is not suitable for the current simulations due to the numerous secondary waves in the material that arise due to reflections from the piston and the pore surfaces, which would severely complicate analysis and interpretation of the results.

\section{Conclusions}

The shock-induced collapse of a $35.0 \mathrm{~nm}$ diameter cylindrical pore in the energetic crystal $\alpha$-RDX was observed for three different impact velocities using MD simulations. For the $1.0 \mathrm{~km} \mathrm{~s}^{-1}$ shock, the pore collapses in a viscoplastic manner wherein net translational motion ceases after collapse and there is little convection of heat to material away from the collapse zone. The collapse at $2.0 \mathrm{~km} \mathrm{~s}^{-1}$ exhibits more focusing along the pore centerline than does the $1.0 \mathrm{~km} \mathrm{~s}^{-1}$ shock. Material focusing results in a greater amount of downstream wall penetration following impact. The stronger penetration produces more molecular rotation during and after collapse along with a greater amount of energy convection to material away from the initial collapse zone.

For the $3.0 \mathrm{~km} \mathrm{~s}^{-1}$ shock, an enhanced focusing of material occurs along the pore centerline that is sufficient to produce a fluid-like jet structure during collapse. The jet causes a greater deposition of mechanical energy at the 
downstream wall leading to much higher temperatures achieved in the collapse zone. Jet penetration into the downstream wall upon impact leads to the development of a following flow after closure. Translational kinetic energy in this inflow region is redistributed to intramolecular kinetic energy and is spread to surrounding material through convection. Critical-size hot spots remain beyond the current computational capabilities of MD simulations. However, simulations on the atomic scale such as those presented in this work can provide insight into the fundamental collapse mechanism of pores in energetic materials. This information, in combination with continuum-scale models and experimental data, can aid the development of a more complete understanding of the role of pore collapse in hot spot generation.

Acknowledgments This work was supported by the U. S. Defense Threat Reduction Agency under Grant No. HDTRA1-10-1-0078 and by the U. S. Air Force Office of Scientific Research under Grant No. FA9550-14-1-0360.

\section{References}

1. Bowden FP, Yoffe AD (1952) Initiation and growth of explosion in liquids and solids. Cambridge University Press, Cambridge

2. Handley C (2011) Numerical modeling of two HMX-based plastic-bonded explosives at the mesoscale. Dissertation, St. Andrews University

3. Tarver CM, Chidester SK, Nichols AL (1996) Critical conditions for impact- and shock-induced hot spots in solid explosives. J Phys Chem 100(14):5794-5799

4. Field JE (1992) Hot spot ignition mechanisms for explosives. Acc Chem Res 25(11):489-496

5. Bowden FP, McOnie MP (1967) Formation of cavities and microjets in liquids and their role in initiation and growth of explosion. Proc R Soc Lond A Mat 298(1452):38-50

6. Coley GD, Field JE (1973) The role of cavities in the initiation and growth of explosion in liquids. Proc R Soc Lond A Mat 335(1600):67-86

7. Winter RE, Field JE (1975) The role of localized plastic flow in the impact initiation of explosives. Proc R Soc Lond A Mat 343(1634):399-413

8. Coffey CS (1981) Phonon generation and energy localization by moving edge dislocations. Phys Rev B 24(12):6984-6990

9. Bowden FP, Gurton OA (1949) Initiation of solid explosives by impact and friction: the influence of grit. Proc R Soc Lond A Mat 198(1054):337-349

10. Dear JP, Field JE, Walton AJ (1988) Gas compression and jet formation in cavities collapsed by a shock wave. Nature 332(6164):505-508

11. Bourne NK, Field JE (1991) Bubble collapse and the initiation of explosion. Proc R Soc Lond A Mat 435(1894):423-435

12. Bourne NK, Field JE (1999) Explosive ignition by the collapse of cavities. Proc R Soc Lond A Mat 455(1987):2411-2426

13. Bourne NK, Milne AM (2003) The temperature of a shock-collapsed cavity. Proc R Soc Lond A Mat 459(2036):1851-1861

14. Swantek AB, Austin JM (2010) Collapse of void arrays under stress wave loading. J Fluid Mech 649:399-427

15. Dattelbaum DM, Sheffield SA, Stahl DB, Dattelbaum AM, Trott $\mathrm{W}$, Engelke R (2010) Influence of hot spot features on the initiation characteristics of heterogeneous nitromethane. In: Fourteenth Symposium (International) on Detonation

16. Stoltz CA, Mason BP, Hooper J (2010) Neutron scattering study of internal void structure in RDX. J Appl Phys 107(10):103527

17. Mang JT, Hjelm RP, Francois EG (2010) Measurement of porosity in a composite high explosive as a function of pressing conditions by ultra-small-angle neutron scattering with contrast variation. Propell Explos Pyrot 35(1):7-14

18. Mang JT, Hjelm RP (2011) Small-angle neutron scattering and contrast variation measurement of the interfacial surface area in PBX 9501 as a function of pressing intensity. Propell Explos Pyrot 36(5):439-445

19. Menikoff R (2004) Pore collapse and hot spots in HMX. AIP Conf Proc 706(1):393-396

20. Barton NR, Winter NW, Reaugh JE (2009) Defect evolution and pore collapse in crystalline energetic materials. Model Simul Mater Sci 17(3):035003

21. Austin RA, Barton NR, Howard WM, Fried LE (2014) Modeling pore collapse and chemical reactions in shock-loaded HMX crystals. J Phys 500(5):052002

22. Austin RA, Barton NR, Reaugh JE, Fried LE (2015) Direct numerical simulation of shear localization and decomposition reactions in shock-loaded HMX crystal. J Appl Phys 117(18):185902

23. Ball GJ, Howell BP, Leighton TG, Schofield MJ (2000) Shockinduced collapse of a cylindrical air cavity in water: a free-Lagrange simulation. Shock Waves 10(4):265-276

24. Bourne NK, Field JE (1992) Shock-induced collapse of single cavities in liquids. J Fluid Mech 244:225-240

25. Tran L, Udaykumar HS (2006) Simulation of void collapse in an energetic material, Part 1: inert case. J Propul Power 22(5):947-958

26. Tran L, Udaykumar HS (2006) Simulation of void collapse in an energetic material, Part 2: reactive case. J Propul Power 22(5):959-974

27. Jackson TL, Buckmaster JD, Zhang J, Anderson MJ (2015) Pore collapse in an energetic material from the micro-scale to the macro-scale. Combust Theor Model 19(3):347-381

28. Levesque GA, Vitello P (2015) The effect of pore morphology on hot spot temperature. Propell Explos Pyrot 40(2):303-308

29. Holian BL, Germann TC, Maillet J-B, White CT (2002) Atomistic mechanism for hot spot initiation. Phys Rev Lett $89(28): 285501$

30. Hatano T (2004) Spatiotemporal behavior of void collapse in shocked solids. Phys Rev Lett 92(1):015503

31. Shi Y, Brenner DW (2008) Jetting and detonation initiation in shock induced collapse of nanometer-scale voids. J Phys Chem C 112(16):6263-6270

32. Mintmire JW, Robertson DH, White CT (1994) Molecular-dynamics simulations of void collapse in shocked model-molecular solids. Phys Rev B 49(21):14859-14864

33. Herring SD, Germann TC, Grønbech-Jensen N (2010) Effects of void size, density, and arrangement on deflagration and detonation sensitivity of a reactive empirical bond order high explosive. Phys Rev B 82(21):214108

34. van Duin ACT, Dasgupta S, Lorant F, Goddard WA III (2001) ReaxFF: a reactive force field for hydrocarbons. J Phys Chem A 105(41):9396-9409

35. Strachan A, Kober EM, van Duin ACT, Oxgaard J, Goddard WA III (2005) Thermal decomposition of RDX from reactive molecular dynamics. J Chem Phys 122(5):054502-054510

36. Shan TR, Thompson AP (2014) Shock-induced hotspot formation and chemical reaction initiation in PETN containing a spherical void. J Phys 500(17):172009

37. Thompson AP, Shan TR (2014) Reactive atomistic simulations of shock-induced initiation processes in mixtures of ammonium nitrate and fuel oil. J Phys 500(5):052046 
38. Hooks DE, Ramos KJ, Martinez AR (2006) Elastic-plastic shock wave profiles in oriented single crystals of cyclotrimethylene trinitramine (RDX) at 2.25 GPa. J Appl Phys 100(2):0249081-024908-7

39. Ramos KJ, Hooks DE, Bahr DF (2009) Direct observation of plasticity and quantitative hardness measurements in single crystal cyclotrimethylene trinitramine by nanoindentation. Philos Mag 89(27):2381-2402

40. Dang NC, Dreger ZA, Gupta YM, Hooks DE (2010) Time-resolved spectroscopic measurements of shock-wave induced decomposition in cyclotrimethylene trinitramine (RDX) crystals: anisotropic response. J Phys Chem A 114(43):11560-11566

41. Ramos KJ, Hooks DE, Sewell TD, Cawkwell MJ (2010) Anomalous hardening under shock compression in (021)-oriented cyclotrimethylene trinitramine single crystals. J Appl Phys 108(6):066105-1-066105-3

42. Doherty RM, Watt DS (2008) Relationship between RDX properties and sensitivity. Propell Explos Pyrot 33(1):4-13

43. Infante-Castillo R, Pacheco-Londoño LC, Hernández-Rivera SP (2010) Monitoring the $\alpha \rightarrow \beta$ solid-solid phase transition of RDX with Raman spectroscopy: a theoretical and experimental study. J Mol Struct 970(1-3):51-58

44. Elban WL, Hoffsommer JC, Armstrong RW (1984) X-ray orientation and hardness experiments on RDX explosive crystals. J Mater Sci 19(2):552-566

45. Patterson JE, Dreger ZA, Gupta YM (2007) Shock wave-induced phase transition in RDX single crystals. J Phys Chem B 111(37):10897-10904

46. Shi Y, Brenner DW (2008) Molecular simulation of the influence of interface faceting on the shock sensitivity of a model plastic bonded explosive. J Phys Chem B 112(47):14898-14904

47. Cawkwell MJ, Sewell TD, Zheng L, Thompson DL (2008) Shock-induced shear bands in an energetic molecular crystal: application of shock-front absorbing boundary conditions to molecular dynamics simulations. Phys Rev B 78(1):014107

48. Bedrov D, Hooper JB, Smith GD, Sewell TD (2009) Shock-induced transformations in crystalline RDX: a uniaxial constantstress Hugoniostat molecular dynamics simulation study. J Chem Phys 131(3):034712

49. Cawkwell MJ, Ramos KJ, Hooks DE, Sewell TD (2010) Homogeneous dislocation nucleation in cyclotrimethylene trinitramine under shock loading. J Appl Phys 107(6):063511-063512

50. Mathew N, Picu RC (2011) Molecular conformational stability in cyclotrimethylene trinitramine crystals. J Chem Phys 135(2): 024510

51. Munday LB, Chung PW, Rice BM, Solares SD (2011) Simulations of high-pressure phases in RDX. J Phys Chem B 115(15): $4378-4386$

52. Nomura K, Kalia RK, Nakano A, Vashishta P (2007) Reactive nanojets: nanostructure-enhanced chemical reactions in a defected energetic crystal. Appl Phys Lett 91(18):183109

53. Choi CS, Prince E (1972) The crystal structure of cyclotrimethylenetrinitramine. Acta Crystallallogr B 28(9):2857-2862

54. Millar DIA, Oswald IDH, Francis DJ, Marshall WG, Pulham CR, Cumming AS (2009) The crystal structure of $\beta$-RDX-an elusive form of an explosive revealed. Chem Commun 5:562-564

55. Millar DIA, Oswald IDH, Barry C, Francis DJ, Marshall WG, Pulham CR, Cumming AS (2010) Pressure-cooking of explosives-the crystal structure of $\varepsilon$-RDX as determined by X-ray and neutron diffraction. Chem Commun 46(31):5662-5664

56. Davidson AJ, Oswald IDH, Francis DJ, Lennie AR, Marshall WG, Millar DIA, Pulham CR, Warren JE, Cumming AS (2008) Explosives under pressure-the crystal structure of $\gamma$-RDX as determined by high-pressure X-ray and neutron diffraction. Cryst Eng Comm 10(2):162-165

57. Munday LB, Solares SD, Chung PW (2012) Generalized stacking fault energy surfaces in the molecular crystal $\alpha$ RDX. Philos Mag 92(24):3036-3050

58. Gallagher HG, Halfpenny PJ, Miller JC, Sherwood JN, Tabor D (1992) Dislocation slip systems in pentaerythritol tetranitrate (PETN) and cyclotrimethylene trinitramine (RDX). Philos Trans R Soc A 339(1654):293-303

59. Mathew N, Picu CR, Chung PW (2013) Peierls stress of dislocations in molecular crystal cyclotrimethylene trinitramine. J Phys Chem A 117(25):5326-5334

60. Allen MP, Tildesley DJ (1987) Computer simulation of liquids. Oxford University Press, Oxford

61. Rapaport DC (2004) The art of molecular dynamics simulation. Cambridge University Press, Cambridge

62. Tuckerman ME (2010) Statistical mechanics: Theory and molecular simulation. Oxford University Press, Oxford

63. Bouma RHB, van der Heijden AEDM, Sewell T, Thompson DL (2011) Simulations of deformation processes in energetic materials. In: Awrejcewicz J (ed) Numerical Simulations. Rijeka, InTech, pp 29-58

64. Rice BM, Sewell TD (2008) Equilibrium molecular dynamics simulations. In: Peiris SM, Piermarini GJ (eds) Static compression of energetic materials. Springer, Berlin, p 330

65. Stuart SJ, Tutein AB, Harrison JA (2000) A reactive potential for hydrocarbons with intermolecular interactions. J Chem Phys 112(14):6472-6486

66. Donald WB, Olga AS, Judith AH, Steven JS, Boris N, Susan BS (2002) A second-generation reactive empirical bond order (REBO) potential energy expression for hydrocarbons. J Phys 14(4):783

67. Plimpton S (1995) Fast parallel algorithms for short-range molecular dynamics. J Comput Phys 117(1):1-19

68. Smith GD, Bharadwaj RK (1999) Quantum chemistry based force field for simulations of HMX. J Phys Chem B 103(18):3570 3575

69. Bedrov D, Ayyagari C, Smith G, Sewell T, Menikoff R, Zaug J (2001) Molecular dynamics simulations of HMX crystal polymorphs using a flexible molecule force field. J Comput-Aided Mater 8(2):77-85

70. Hockney RW, Eastwood JW (1981) Computer simulations using particles. McGraw-Hill, New York

71. Bendat JS, Piersol AG (1980) Engineering applications of correlation and spectral analysis. Wiley, New York

72. Eason RM, Sewell TD (2011) Shock-induced inelastic deformation in oriented crystalline pentaerythritol tetranitrate. J Phys Chem C 116(3):2226-2239

73. Bolesta AV, Zheng L, Thompson DL, Sewell TD (2007) Molecular dynamics simulations of shock waves using the absorbing boundary condition: a case study of methane. Phys Rev B 76(22):224108 